\title{
Detección de enfermedades en el sector agrícola utilizando Inteligencia Artificial
}

\author{
Balzhoyt Roldán Ortega, Rajesh Roshan Biswal, Eddy Sánchez DelaCruz \\ Instituto Tecnológico Superior de Misantla, Departamento de Posgrado, \\ Misantla, Veracruz, México \\ balzhoyt@gmail.com, rjshbswl@gmail.com, eddsacx@gmail.com
}

\begin{abstract}
Resumen. El presente artículo, expone varios trabajos de investigación sobre la posibilidad de detectar enfermedades en los cultivos agrícolas, utilizando técnicas de inteligencia artificial. Los autores de dichos trabajos, hacen propuestas de técnicas de obtención de características de las hojas o frutos de las plantas, así como también el uso de algoritmos clasificadores o de agrupación, todo esto con el fin de determinar si una hoja, presenta signos de alguna enfermedad. Al haber diversos tipos de enfermedades y diversas variedades de plantas, los autores hacen propuestas para utilizar el algoritmo que ellos consideran el que obtendrá mejores resultados. Al final concluimos que, sí es posible detectar enfermedades trayendo consigo un beneficio directo para el agricultor que la implemente, y a que un diagnóstico oportuno, daría una respuesta a la enfermedad y por lo tanto reducción del riesgo en pérdidas económicas.
\end{abstract}

Palabras clave: inteligencia artificial, reconocimiento de patrones, enfermedades, plantas.

\section{Detection of Diseases in the Agricultural Sector using Artificial Intelligence}

\begin{abstract}
The present article, exposes several works of investigation on the possibility of detecting diseases in the agricultural crops, using artificial intelligence techniques. The authors of these works, proposed different techniques for obtaining leaf or fruits characteristics of plants, using classification or clustering algorithms, in order to determine whether or not, a leaf showed signs of disease. Depending on the disease and the plant type, adequate algorithms with the optimum results were proposed. It can be concluded that, it is possible to detect leaf diseases, thereby benefitting the farmer directly. Moreover, a timely diagnosis would result in an effective treatment of the disease, thus avoiding the risk of any economic loss as result.
\end{abstract}

Keywords: artificial intelligence, pattern recognition, diseases, plants. 


\section{Introducción}

Es bien sabido que la prevención y el diagnóstico oportuno de cualquier enfermedad nos traerá la ventaja estratégica sobre dicho padecimiento y en la agricultura no es la excepción, pues sabiendo lo que aqueja a un cultivo o planta, se incrementa las posibilidades de éxito en el tratamiento.

En el mundo en desarrollo, más del $80 \%$ de la producción agrícola es generada por pequeños agricultores [1], y los informes de pérdidas de rendimiento de más del $50 \%$ debido a plagas y enfermedades son comunes [2]. Además, la mayor proporción de personas con problemas de pobreza y hambruna (50\%) vive en estas zonas productivas [3], lo que hace que los pequeños agricultores sean un grupo particularmente vulnerable a las interrupciones en el suministro de alimentos derivadas de patógenos.

Existen métodos para determinar las enfermedades de cualquier planta, como llevar muestras de tejido vegetativo a un laboratorio especializado o llevar a un ingeniero agrónomo experto al sitio del cultivo, en cualquiera de los dos métodos, la desventaja radica en el tiempo necesario para obtener los resultados.

Es por eso, que se han considerado el uso de técnicas de visión artificial y reconocimiento de patrones, asícomo algunos algoritmos de clasificación que de forma automática determinen la posible enfermedad, facilitando la tarea de los especialistas para desarrollar su trabajo y que puedan dar con un diagnóstico oportuno para su tratamiento. Y como dice Barbedo [4], las herramientas para el reconocimiento automático de enfermedades de las plantas tienen el potencial de convertirse en una valiosa fuente de información para ayudar a la toma de decisiones en la agricultura.

En la siguiente sección, se muestran las técnicas utilizadas, así como los trabajos de investigación más significativos, que atienden el desafío de la detección de una enfermedad, a partir del análisis de las características presentes en las hojas de los cultivos.

\section{Técnicas y algoritmos empleados}

Para llegar a la clasificación de las enfermedades, los autores se basan en metodologías ya probadas, como se muestra en la figura 1. Adquisición de los datos, preprocesamiento, extracción de características y reconocimiento, son los pasos o procedimientos que se siguen para obtener resultados as ertivos.

Para la adquisición de las imágenes, se hace uso de cámaras digitales para capturar las hojas o las partes donde es visible el daño causado por la enfermedad, así como también conjuntos de imágenes disponibles en la web y que se han puesto a disposición de todo el público sirviendo como base para el entrenamiento del modelo. Para la imágenes adquiridas por cámara, las tomas fueron en ambientes controlados con resoluciones aceptables, aunque es importante mencionar que Bhange [6] utilizó un teléfono móvil, para adquirir las imágenes y hacer un experimento alterno para ver qué tanto afectaba la calidad de las mismas.

Una vez adquiridas las imágenes, pasan al preprocesamineto, en donde se les darán un tratamiento como el escalado, eliminación de ruido, transformación del espacio de color, ecualización de histograma y todo lo que se pueda hacer para maximizar las características. Para cuando pasa al siguiente proceso, la imagen va más limpia, por lo 
tanto, al aplicarle las técnicas de segmentación, éstas separarán los puntos de interés con mejor precisión, obteniendo datos valiosos que serán más descriptivos de la enfermedad. Una vez teniendo las características, lo siguiente es hacer la clasificación, hacer uso de los algoritmos, obtener los resultados y hacer una descripción de los mismos. Es en este paso, cuando se muestra la eficacia del procedimiento, si se ha clasificado de acuerdo a lo esperado.

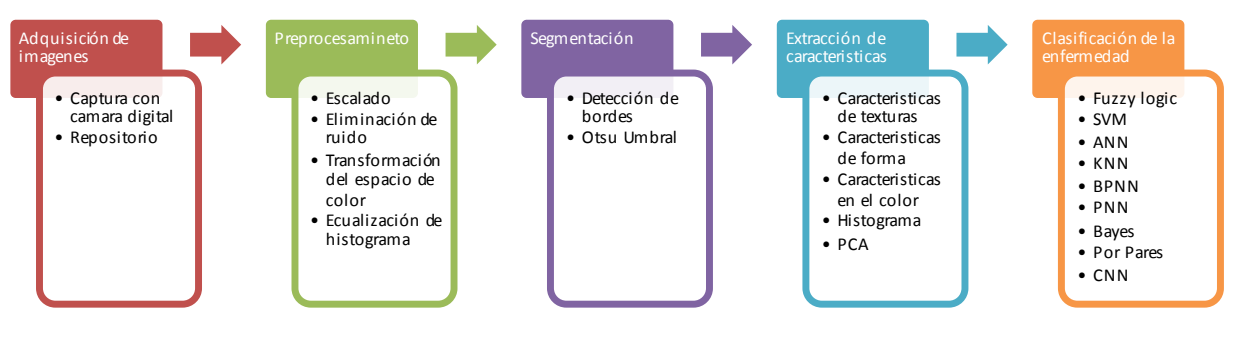

Fig. 1. Metodología generalizada usada en estos artículos.

A continuación, los algoritmos con sus particularidades para ser elegidos como clasificadores en estos estudios.

Tabla 1. Algoritmos y sus características.

\begin{tabular}{|c|c|}
\hline Algoritmo & Propiedades \\
\hline Fuzzy logic & $\begin{array}{l}\text { Se basa en reglas heurísticas, se usa para procesos altamente no } \\
\text { lineales [5]. Fácil implementación. }\end{array}$ \\
\hline SVM & $\begin{array}{l}\text { Busca un hiperplano que funciona como separador. En el } \\
\text { entrenamiento y problemas típicos es muy eficiente [6]. }\end{array}$ \\
\hline Bayes & $\begin{array}{l}\text { Es muy eficiente donde se utiliza este tipo de entorno de } \\
\text { aprendizaje supervisado. No se requiere de gran cantidad de datos } \\
\text { para su entrenamiento [7]. }\end{array}$ \\
\hline $\mathrm{KNN}$ & $\begin{array}{l}\text { Busca de las observaciones más cercanas a la que está tratando de } \\
\text { predecir y clasifica el punto de interés basado en la may oría de los } \\
\text { datos de entrenamiento.[10] }\end{array}$ \\
\hline ANN & $\begin{array}{l}\text { La clasificación es muy eficiente, a costa de un entrenamiento } \\
\text { costoso computacionalmente.[11] }\end{array}$ \\
\hline $\mathrm{CNN}$ & $\begin{array}{l}\text { El desempeño de las redes neuronales convolucionales en el } \\
\text { reconocimiento de objetos y la clasificación de imágenes ha hecho } \\
\text { un gran progreso en los últimos años. Tienden a ser más precisas a } \\
\text { costa del alto costo computacional. En el entrenamiento puede } \\
\text { requerir un número considerable de imágenes para producir } \\
\text { resultados fiables [15] }\end{array}$ \\
\hline
\end{tabular}

En la siguiente sección, se muestra los trabajos significativos, relacionados con la detección de las enfermedades en varios tipos de plantas, utilizando diversos algoritmos de aprendizaje automático, pero siempre siguiendo la mis ma metodología. 


\section{Revisión de la literatura}

Derwin Suhartono et al. (2013) utilizaron un sistema de lógica difusa y árboles de decisión y con la ayuda de un experto humano, pudieron hacer un reconocimiento de las enfermedades del café, donde obtuvieron las características de los síntomas que se presentan en la planta y así poder hacer su árbol de decisión. Los resultados que obtuvieron están dentro del $85 \%$ de exactitud. Aunque ésta investigación no trata de técnicas de visión artificial, se puede notar que se ha dado un paso muy importante para la detección de enfermedades, utilizando sistemas expertos aplicando arboles de decisión con lógica difusa [5].

Bhange y Hingoliwala (2015) proponen una herramienta web, donde los agricultores que cultivan la granada, subirán una imagen del fruto para ser analizada y con el modelo entrenado se puede comprobar si está o no infectado el fruto. La técnica utilizada se basa en extraer las características de las imágenes de la granada, como el color, morfología y el vector de coherencia de color para posteriormente realizar la clasificación mediante el algoritmo SVM dándoles una precisión del 85\% con una cámara de 10 megapíxeles. Los autores sabiendo que los agricultores no siempre tendrán los medios óptimos de captura para subir las imágenes al sistema, realizaron pruebas significativas con las cámaras de los dispositivos móviles con resoluciones de 5 y 3 megapíxeles, y obtuvieron resultados de $82 \%$ y $79 \%$ respectivamente [6].

Mengistu et al. (2016) utilizaron una ANN, KNN, Naive Bayes, un hibrido de mapas auto organizables (SOM) y función de base radial (RBF), todo esto para poder determinar el mejor algoritmo que clasificara las enfermedades de roya, marchitamiento del café (CWD) y la CFD que afecta al fruto del café. En su trabajo exponen que consiguieron para el KNN 58.16\%, para Naive Bayes obtuvieron $53.47 \%$, para ANN 79.04\%, y para la combinación de RBF y SOM obtienen $90.07 \%$ de exactitud, lo cual muestra una gran mejora con respecto a los anteriores algoritmos, aunque dejan nota que éste último, les lleva más tiempo en el entrenamiento [7].

Singh y Misra (2017), realizaron pruebas para obtener reconocimiento de enfermedades o quemaduras presentadas en las hojas, Los cultivos de estudio fueron de plátano, frijol, limón y rosas. Después de procesar las imágenes, Proponen hacer la segmentación mediante algoritmos genéticos, y hacer el agrupamiento. Para la extracción de características utilizaron el método de concurrencia de color, ya que consideran mejor utilizar la imagen a color que la tradicional escala de grises. Para hacer la clasificación, utilizaron MDC con K-Mean obteniendo $86.54 \%$, MDC con un algoritmo propuesto porellos, obteniendo una mejora de $93.63 \%$ y SVM con algoritmo propuesto obteniendo una mejora significativa de $95.71 \%$. Todos estos porcentajes describiendo un promedio general de los cuatro cultivos de estudio [8]

Barbedo et al. (2016) proponen algoritmos para identificar múltiples enfermedades de las plantas, basándose en el análisis del color y usando un algoritmo de clasificación por pares. Según ellos con su metodología, les permite operar en condiciones no controladas y así poder abarcar un gran número de enfermedades. Este método fue probado con un gran conjunto, sin restricciones de las imágenes de las hojas que contienen los síntomas que pertenecen a 74 enfermedades, 4 plagas y 4 trastomos abióticos, que afecta a 12 especies de plantas diferentes. Los resultados obtenidos fueron entre $40 \%$ y $80 \%$ de precisión [9] 
Qin et al. (2016), nos dicen que, encontraron una solución factible para el diagnóstico e identificación de cuatro enfermedades de la alfalfa. Extrajeron 129 características de textura, color y forma de las 1651 imágenes utilizando los métodos ReliefF, 1R y CFS. Para clasificar las enfermedades, utilizaron SVM, KNN y Random Forest. obteniendo que el mejor clasificador fue SVM y el método RelifF para la obtención de característica, ya que consiguieron $97.64 \%$ en precisión para el conjunto de entrenamiento y $94.74 \%$ para el conjunto de pruebas [10].

Pujari et al. (2016) compararon los algoritmos SVM y ANN para clasificar enfermedades en diversos cultivos que han sido atacados por hongos, bacterias, nematodos y deficiencia de nutrientes. Mencionan que los síntomas de las enfermedades de las plantas exhiben diferentes propiedades como el color, forma y textura y en base a esto, se obtiene las características. Consideran el color como una dimensión importante, pero aplicando reducción de dimensionalidad descubren por experimentación que, de 24 solo 8 características son significativas para la clasificación de las enfermedades. Al final ponen a prueba los dos algoritmos y encuentran que con SVM obtienen $92.17 \%$ de precisión y que con ANN solo $87.48 \%$ por lo cual demuestran que, para este caso, el SVM es mejor clasificador [11].

Kiani y Mamedov (2017) tratan mediante su trabajo, de probar que no es necesario utilizar una red neuronal o algoritmo complejo para verificar si existe o no una enfermedad característica en las hojas de las fresas. Para los autores les es mejor usar algoritmos de lógica difusa para la clasificación que cualquier otro método de que consuma más recurso computacional como las redes neuronales. Los res ultados que obtienen son alentadores, ya que alcanzaron una optimización del $97 \%$ en la segmentación y clasificación de las enfermedades con un tiempo de procesamiento de 1.2 segundos. Con este tipo de técnicas comprueban que es factible de ser implantadas en un circuito integrado, adaptado a un robot con visión artificial y que pudiera hacer una inspección automatizada en un centro botánico moderno [12].

Mohanty et al. (2016) realizó 60 experimentos donde usa redes neuronales convolucionales profunda para identificar 14 especies de cultivos y 26 enfermedades, utilizando modelos entrenados como AlexNet [17] y GoogleNet [18]. Utilizan el enfoque de Krizhevsky et al.[20] que demuestra por primera vez que la capacitación supervisada de extremo a extremo con una arquitectura CNN es una posible opción para un gran número de clases, superando el enfoque tradicional de utilizar características diseñadas a mano. Dentro del conjunto de datos de PlantVillage de 54,306 imágenes que contienen 38 clases de 14 especies de cultivos y 26 enfermedades (o su ausencia), este objetivo se ha logrado, como lo demuestra la precisión máxima del $99.35 \%$. Por lo tanto, sin ningún tipo de ingeniería de características, el modelo clasifica correctamente los cultivos y las enfermedades de 38 clases posibles en 993 de cada 1000 imágenes. Los autores notaron que para el entrenamiento se requiere mucho trabajo computacional pero para la clasificación es menos de un segundo, creen que se podría implementar en un teléfono celular [13].

Ashqar y Abu-Naser (2019) realizaron un estudio que consistió en analizar 9,000 imágenes de hojas de tomate, para producir un modelo que se pudiera utilizar en teléfonos inteligentes, con el propósito de identificar 5 tipos de enfermedades. Su modelo estaría basado en una red convolucional profunda, pero que estaría conformada por dos partes, La primera parte del modelo (las funciones de extracción), que era el mismo para enfoque a todo color y enfoque de escala de grises, que consta de 4 capas 
convolucionales con función de activación Relu, cada una seguida por capa Max Pooling y la segunda parte contendría dos capas densas para contener los dos enfoques, color y escala de grises. Al final, demostraron que trabajar con las características a color, les da mejores resultados $99.84 \%$ con respecto a escala de grises con un $95.54 \%$ [14].

Barbedo (2019) ha estado trabajando con redes neuronales convolucionales de varios cultivos, buscando niveles de enfermedad en las plantas, pudiendo clasificar a cultivos sanos con un $89 \%$ de exactitud, ligeramente enfermos con un $31 \%$, moderadamente enfermos $87 \%$ y gravemente enfermos con un 94\%. Aunque los resultados son satisfactorios, concluye que la clasificación de las enfermedades de las plantas a partir de imágenes digitales, es muy difícil. Por otra parte, las limitaciones del conjunto de datos en términos de cantidad y variedad de muestras, siguen impidiendo la aparición de sistemas verdaderamente integrales para hacer funciones de clasificación de enfermedades [15].

Khan at al (2019) propone el uso de VGGNet [19] y AlexNetOWTBn [20] como arquitecturas de una red convolucional profunda, para automatizar el proceso de clasificación de las enfermedades que se presentan en las hojas de tomate. Las enfermedades a clasificar fueron el tizón temprano, oidio y mildiu. Las imágenes recogidas son pre-procesadas utilizando técnicas de procesamiento de imágenes como la reducción de ruido, regresión y mejora de procesamiento de imágenes que ayuda en la reducción de costes y tiempo de cálculo. Posteriormente extrajo las características del conjunto de datos utilizando mapas de convolución donde se aplicaban a los datos de entrada, imágenes de hojas sana e infectadas. Aunque la arquitectura ha presentado resultados muy precisos, para este estudio solo obtuvieron $32.23 \%$ usando la arquitectura AlexNetOWTBn y 33.27\% para VGG [16].

En la tabla 2 se muestra el resumen de los resultados obtenidos por las investigaciones expuestas.

Tabla 2. Resumen de los algoritmos y sus resultados.

\begin{tabular}{|c|c|c|c|}
\hline Referencia. & Cultivo & Clasificador & $\begin{array}{l}\text { Precisión en los } \\
\text { resultados }\end{array}$ \\
\hline [5] & Café & Fuzzy logic & $85.00 \%$ \\
\hline [6] & Granada & SVM & $82.00 \%$ \\
\hline \multirow{4}{*}{ [7] } & \multirow{4}{*}{ Café } & KNN & $58.16 \%$ \\
\hline & & ANN & $79.04 \%$ \\
\hline & & N. BAYES & $53.47 \%$ \\
\hline & & RBF y SOM & $90.07 \%$ \\
\hline \multirow{3}{*}[8]{} & \multirow{3}{*}{$\begin{array}{l}\text { Plátano, Frijol, Limón, } \\
\text { Rosa }\end{array}$} & MDC + K-M eans & $86.54 \%$ \\
\hline & & MDC + propuesta & $93.63 \%$ \\
\hline & & SVM + propuesta & $95.71 \%$ \\
\hline \multirow{8}{*}{ [9] } & Frijol & \multirow{8}{*}{$\begin{array}{l}\text { Propuesta Clasificación } \\
\text { por pares }\end{array}$} & $50.00 \%$ \\
\hline & Mandioca & & $46.00 \%$ \\
\hline & Agrios & & $56.00 \%$ \\
\hline & Árbol de coco & & $71.00 \%$ \\
\hline & Café & & $53.00 \%$ \\
\hline & Maíz & & $40.00 \%$ \\
\hline & Algodón & & $76.00 \%$ \\
\hline & Uva & & $58.00 \%$ \\
\hline
\end{tabular}


Detección de enfermedades en el sector agrícola utilizando Inteligencia Artificial

\begin{tabular}{|c|c|c|c|}
\hline Referencia. & Cultivo & Clasificador & $\begin{array}{l}\text { Precisión en los } \\
\text { resultados }\end{array}$ \\
\hline & Maracuyá & & $56.00 \%$ \\
\hline & Soja & & $58.00 \%$ \\
\hline & Caña de Azúcar & & $59.00 \%$ \\
\hline & Trigo & & $70.00 \%$ \\
\hline$[10]$ & Alfalfa & SVM & $94.74 \%$ \\
\hline & Trigo, girasol, uva, & ANN & $87.48 \%$ \\
\hline [11] & $\begin{array}{l}\text { maíz, pepino, algodón, } \\
\text { col, tomate }\end{array}$ & SVM & $92.17 \%$ \\
\hline [12] & Fresa & Fuzzy logic & $97.00 \%$ \\
\hline [13] & Varios & $\mathrm{CNN}$ & $99.35 \%$ \\
\hline [14] & Tomate & CNN & $99.84 \%$ \\
\hline \multirow{15}{*}{ [15] } & Frijol & & $95.00 \%$ \\
\hline & Mandioca & & $83.00 \%$ \\
\hline & Naranjo & & $62.00 \%$ \\
\hline & Coco & & $97.00 \%$ \\
\hline & Maíz & & $66.00 \%$ \\
\hline & Café & & $77.00 \%$ \\
\hline & Algodón & & $100 \%$ \\
\hline & Anacardo & & $83.00 \%$ \\
\hline & Uva & & $81.00 \%$ \\
\hline & Col rizada & & $100 \%$ \\
\hline & Maracuyá & & $90.00 \%$ \\
\hline & Haba de soja & & $76.00 \%$ \\
\hline & Caña de azúcar & & $100 \%$ \\
\hline & Trigo & & $61.00 \%$ \\
\hline & Total & & $82.00 \%$ \\
\hline [16] & Tomate & $\mathrm{CNN}$ & $33.27 \%$ \\
\hline
\end{tabular}

\section{Discusión y resultados}

Una de las ventajas que podemos observar cuando Derwin Suhartono et al. (2013) [5] utilizó el algoritmo clasificador de lógica difusa, es lo fácil y rápido que pudo implementarlo, pues no requiere de una gran colección de datos para su entrenamiento, y le presentó resultados más o menos favorables al momento de querer clasificar . Ahora que, si se disminuyen la clase objetivo, pues tendría mejores resultados, como lo demuestra el trabajo de Kiani y Mamedov (2017) [12], que consiguió mejor precisión por solo el hecho de predecir solo una enfermedad.

La propuesta que hace Mengistu et al. (2016) [7], con utilizar RBF y SOM parece bastante buena, pues son las que les dio mejores resultados, comparados con KNN, ANN, Bayes, y esto se debe a que su enfoque de obtener las características por su textura y color, hizo que tuviera mejores resultados, pues la elección de esta última es la que mejor se desenvuelve para estos casos.

De los algoritmos SVM se puede concluir que es otra buena alternativa para la resolución de estos casos, aunque en un principio, no fueron pensados para resolver este tipo de problemas, con el paso del tiempo se han ido adaptando y presentando resultados muy buenos, como los de Pujari et al. (2016) [11], Singh y Misra (2017) [8] y Bhange y Hingoliwala (2015) [6]. 
Cuando la clasificación es para pocas enfermedades, se podría decir que es relativamente fácil, pero cuando realmente se quiere abarcar más enfermedades o más cultivos, es cuando aumenta la complejidad, y una propuesta de solución es la que presenta Barbedo et al. (2016) [9], con su clasificación por pares donde se basa en la teoría de que las plantas presentan síntomas parecidos cuando son atacados por la misma enfermedad y que su algoritmo puede ser reentrenado para nuevas enfermedades.

Otra propuesta para las mismas plantas y las mismas enfermedades, fue presentada por el mismo Barbedo [15] pero en pero en el año 2019 al utilizar redes neuronales convolucionales, declara que es la mejor forma para hacer este tipo de estudios, y que la única limitante hoy en día, es la disponibilidad de grandes cantidades de datos para hacer un buen entrenamiento de la red.

\section{Conclusión}

Como se pudo observar en esta revisión de artículos, los trabajos que obtuvieron mejores resultados son redes neuronales convolucionales, su uso es cada vez más tendencioso para este tipo de problemáticas, pues se obtienen diagnósticos más cercanos a lo que un experto humano determinaría. El único problema sería que el entrenamiento es demasiado costoso computacionalmente y se requiere una gran cantidad de datos para hacerlo.

Si los datos de estudio no fuesen suficientes, la recomendación sería arboles de decisión con lógica difusa, ya que es el algoritmo con mejores resultados después de las redes convolucionales. Aunque no es muy preciso, podemos confiar que a medida que avancen las tecnologías, se podrá llegar a valores más óptimos y con un costo menor computacionalmente.

Otro punto importante que podemos hacer notar es que, con el uso de nuevas tecnologías como el aprendizaje automático y el reconocimiento de patrones, se pueden detectar enfermedades en los cultivos y hacer un diagnóstico oportuno, dis minuyendo el riesgo de pérdidas agrícolas y económicas, lo que traería un beneficio directo a los agricultores que la implementase.

\section{Referencias}

1. Tai, A.P., Martin, M.V., Heald, C.L.: Threat to future global food security from climate change and ozone air pollution. Nature Climate Change 4(9), 817 (2014)

2. Harvey, C.A., Rakotobe, Z.L., Rao, N.S., Dave, R., Razafimahatratra, H., Rabarijohn, R.H., MacKinnon, J.L.: Extreme vulnerability of smallholder farmers to agricultural risks and climate change in Madagascar. Philosophical Transactions of the Royal Society B: Biological Sciences 369(1639), 20130089 (2014)

3. Sanchez, P.A., Swaminathan, M.S.: Cutting world hunger in half. Science 307(5708), 357359 (2005)

4. Barbedo, J.G.A.: Digital image processingtechniques for detecting, quantify ing and classifying plant diseases. SpringerPlus 2(1), 660 (2013)

5. Suhartono, D., Aditya, W., Lestari, M., Yasin, M.: Expert system in detecting coffee plant diseases. Int. J. Electr. Energy 1(3), 156-162 (2013) 
6. Bhange, M., Hingoliwala, H.A.: Smart farming: Pomegranate disease detection using image processing. Procedia Computer Science 58, 280-288 (2015)

7. Mengistu, A.D., Alemay ehu, D.M., Mengistu, S.G.: Ethiopian coffee plant diseases recognition based on imaging and machine learning techniques. International Journal of Database Theory and Application 9(4), 79-88 (2016)

8. Singh, V., Misra, A.K.: Detection of plant leaf diseases using image segmentation and soft computing techniques. Information processing in Agriculture 4(1), 41-49 (2017)

9. Barbedo, J.G.A., Koenigkan, L.V., Santos, T.T.: Identifying multiple plant diseases using digital image processing. Biosystems engineering, vol. 147, pp. 104-116 (2016)

10. Qin, F., Liu, D., Sun, B., Ruan, L., Ma, Z., Wang, H.: Identification of alfalfa leaf diseases using image recognition technology. PloS one 11(12), e0168274 (2016)

11. Pujari, D., Yakkundimath, R., Byadgi, A.S.: SVM and ANN based classification of plant diseases using feature reduction technique. IJIM AI 3(7), 6-14 (2016)

12. Kiani, E., Mamedov, T.: Identification of plant disease infection using soft-computing: Application to modern botany. Procedia computer science, vol. 120, pp. 893-900 (2017)

13. Mohanty, S.P., Hughes, D.P., Salathé, M.: Using deep learning for image-based plant disease detection. Frontiers in plant science, 7, 1419 (2016)

14. Ashqar, B.A., Abu-Naser, S.S.: Image-Based Tomato Leaves Diseases Detection Using Deep Learning (2019)

15. Barbedo, J.G.A.: Plant disease identification from individual lesions and spots using deep learning. Biosy stems Engineering, 180, pp. 96-107 (2019)

16. Khan, S., Shaikh, A.A., Ansari, H., Ansari, N.: Disorder Detection in Tomato Plant Using Deep Learning. In: Ayesha, A., Ansari, H., Ansari, N. (eds.), Disorder Detection in Tomato Plant Using Deep Learning (February 24, 2019) (2019)

17. Krizhevskv. A.. Sutskever. I.. Hinton. G.E.: Imagenet classification with deep convolutional neural networks. Advances in neural information processing systems, pp. 1097-1105 (2012)

18. Szegedy, C., Liu, W., Jia, Y., Sermanet, P., Reed, S., Anguelov, D., Rabinovich, A.: Going deeper with convolutions. In: Proceedings of the IEEE conference on computer vision and pattern recognition, pp. 1-9 (2015)

19. Simonyan, K., Zisserman, A.: Very deep convolutional networks for large-scale image recognition. arXiv preprint arXiv:1409.1556 (2014)

20. Krizhevsky, A.: One weird trick for parallelizing convolutional neural networks. arXiv preprint arXiv:1404.5997 (2014) 